\title{
Directives concernant les «directives anticipées»: version abrégée et questions types relatives à l'échelle des valeurs
}

Académie Suisse

des Sciences Médicales

(ASSM)
D’une manière générale, les textes qui suivent incluent toujours les personnes de sexe féminin.
La «praticabilité au quotidien» des directives est un facteur primordial pour leur application dans la pratique; c'est ce qui ressort non seulement des différentes prises de position exprimées lors des procédures de consultations des directives de l'Académie Suisse des Sciences Médicales (ASSM), mais également des résultats d'une évaluation des directives de l'ASSM. A cet égard, le volume d'une directive joue un rôle important; selon le thème, les directives sont plus ou moins longues, mais on constate néanmoins, ces dernières années, une tendance à rédiger des directives plus longues. La moitié des personnes sondées jugent les directives de l'ASSM importantes, mais n'ont pas assez de temps pour les étudier. Des versions abrégées donnant un aperçu rapide des principaux points pourraient pallier cet inconvénient. A cette occasion, l'ASSM a publié pour la première fois une version abrégée des directives concernant les «Directives anticipées»; en même temps, elle a élaboré des «questions types concernant l'échelle des valeurs». La version abrégée ne remplace pas la version intégrale des directives, mais donne des indications pour la pratique. Les renvois aux différents chapitres se réfèrent aux directives.

\section{Instructions relatives} aux «Directives anticipées»

Les directives anticipées consignent à l'avance la volonté d'un patient* en cas d'incapacité de discernement; elles peuvent se rapporter à différents domaines d'application. A cet égard, les tâches les plus diverses incombent aux médecins, soignants et autres professionnels de la santé: ils assurent l'information de base relative aux directives anticipées, abordent le sujet avec le patient au moment opportun, lui font part des exigences concernant le contenu et la forme des directives anticipées, et lui apportent une aide au moment de la rédaction ou vérifient l'actualité de directives existantes. Il est important que l'entretien concernant les directives anticipées se déroule d'une façon qui soit adaptée au patient et que ses angoisses par rapport à certaines maladies ou mesures de soin, ses expériences négatives avec certains traitements ou encore ses craintes soient abordées.

Si un patient n'est plus capable de discernement, l'équipe soignante a le devoir d'appliquer les dispositions formulées dans les directives anticipées lors d'une situation thérapeutique concrète, dans la mesure où celle-ci est prévue dans les directives anticipées.
Il n'est pas nécessaire que tous les médecins et soignants soient des «spécialistes» des directives anticipées. Toutefois, dans les grandes institutions, il serait utile qu'un certain nombre de collaborateurs disposent des connaissances nécessaires qui leur permettent de répondre aux questions des professionnels de la santé ou des patients et, le cas échéant, de les conseiller. Il existe en outre des organisations qui soutiennent les médecins, soignants et auteurs de directives anticipées lors de leur rédaction.

Les directives anticipées sont-elles contraignantes? (chapitres 2 et 3 )

A ce jour, ce n'est qu'au niveau cantonal - et seulement dans certains cantons - que des réglementions légales concernant les directives anticipées ont été instaurées. Avec l'entrée en vigueur de la nouvelle Loi sur la protection des adultes (en 2012 au plus tôt), les directives anticipées seront explicitement ancrées dans le droit fédéral. Selon cette loi, les directives anticipées devront être appliquées, à moins qu'elles n'enfreignent les dispositions légales ou que des indices laissent supposer que le patient a changé d'avis. En principe, la règle suivante est actuellement appliquée: plus les directives anticipées sont claires, plus elles auront de poids lors de la prise de décision.

\section{Qui peut rédiger des directives anticipées} et quels sont les points qui méritent une attention particulière? (chapitres 3.2. à 3.4. et 5.2.2.)

Toute personne capable de discernement peut rédiger des directives anticipées. Ceci vaut également pour des adolescents capables de discernement. La décision de rédiger des directives anticipées doit être prise librement, c'est-à-dire sans contrainte extérieure. Les directives anticipées doivent être rédigées par écrit, datées et signées de la main de l'auteur. Elles peuvent à tout moment être modifiées ou révoquées par écrit ou oralement. Il convient d'informer le patient de l'importance d'actualiser ses directives anticipées (cf. ci-dessus). C'est pourquoi il est recommandé de vérifier régulièrement la validité des directives anticipées. Si l'attitude du patient face à la vie, la maladie et la mort («échelle personnelle des valeurs») ou la santé du patient changent, les directives anticipées devraient être réadaptées. 


\section{Contenus des directives anticipées (chapitre 4)}

- Indications concernant l'identité de I'auteur (nom, prénom, date de naissance)

- Confirmation de la capacité de discernement («En pleine possession de mes moyens et après mûre réflexion, je prends par la présente des décisions à appliquer au cas où, suite à une maladie ou un accident, je ne serais plus capable d'exprimer ma volonté»). Remarque: Dans des situations, où la capacité de discernement pourrait être mise en doute ultérieurement (par exemple en cas de début de démence ou de maladie psychiatrique), afin d'éviter les incertitudes, la capacité de discernement peut, en plus, être confirmée par un médecin ou une tierce personne.

- Description de l'échelle personnelle des valeurs: Que signifie concrètement pour l'auteur la «qualité de vie» et une «mort dans la dignité»? Avant de prendre une décision médicale dans des situations limites, quelles sont les convictions, angoisses et attentes qui doivent être prises en considération? Cf. ciaprès «Questions types concernant l'échelle des valeurs».

- Désignation d'au moins un représentant thérapeutique avec indication de ses coordonnées. Dans l'idéal, un remplaçant est également nommé.

- Précision des situations pour lesquelles les directives anticipées sont rédigées resp. dans quelles situations elles doivent être appliquées.

- Indications relatives aux buts d'un traitement dans des situations données.

- Consentement resp. renoncement à des mesures médicales spécifiques. $\mathrm{Si}$, au moment de la rédaction des directives anticipées, le patient souffre déjà d'une maladie, elle doit être mentionnée dans les directives anticipées et celles-ci devront être adaptées en fonction de cette maladie, de son déroulement probable, des complications possibles et des mesures envisageables.

- Consentement au don d'organes

- Utilisation du corps après la mort (autopsie)

- Date et signature

Toutes les directives anticipées sont-elles valables? (chapitres 3.4 et 9)

En règle générale, les exigences formelles doivent être remplies: forme écrite, capacité de discernement, libre consentement, datation, signature de la main de l'auteur.

La validité des directives anticipées n'est pas limitée dans le temps. Il est important que des directives anticipées reflètent la volonté actuelle du patient. Tant qu'il n'existe aucun signe indiquant que la volonté du patient a changé (indications du représentant thérapeutique, des proches ou encore des médecins, infirmiers et soignants), on peut supposer que les directives anticipées expriment la volonté du patient. Si une attitude non verbale du patient (lorsqu'il s'agit de patients atteints de démence, par exemple) laisse supposer que ses directives anticipées ne correspondent plus à sa volonté actuelle, il convient de les vérifier soigneusement avec le représentant thérapeutique et les proches. La décision devrait faire l'objet d'un consensus non seulement au sein de l'équipe soignante, mais également avec les éventuels représentants thérapeutiques et les proches. Les écarts par rapport au texte des directives anticipées doivent être fondés et consignés dans le dossier de soins du patient (cf. chapitre 9 «Changement de volonté»).

A quel moment des directives anticipées doivent-elles être appliquées et de quelle manière doit-on procéder? (chapitre 8)

Des directives anticipées ne sont appliquées que si le patient est incapable de discernement, c'est-à-dire lorsqu'il ne peut plus exprimer lui-même sa volonté. $\mathrm{Si}$, par contre, le patient est capable de discernement, sa volonté exprimée doit être respectée.

Lorsqu'un patient est incapable de discernement, les points suivants doivent être clarifiés:

- A-t-il rédigé des directives anticipées (recherche des directives ou d'une carte les signalant, questions au médecin de famille ou aux proches)?

- Le patient a-t-il désigné un représentant thérapeutique? Si oui, celui-ci doit être informé et impliqué dans le plan thérapeutique.

Les décisions thérapeutiques prises sur la base des directives anticipées devraient faire l'objet d'un consensus entre l'équipe soignante, les représentants thérapeutiques et les proches.

Les directives anticipées doivent-elles être appliquées même en situation d'urgence? (chapitre 8)

En situation d'urgence, par exemple en cas d'accident de la circulation, il est généralement impossible de vérifier si des directives anticipées ont été rédigées ou non. C'est pourquoi les mesures nécessaires au maintien en vie doivent être introduites. Toutefois, l'existence éventuelle de directives anticipées doit être vérifiée ultérieurement; si elles existent, elles doivent être intégrées dans le plan thérapeutique et les mesures déjà introduites doivent, le cas échéant, être interrompues.

\section{Comment connaît-on l'existence de directives anticipées (chapitre 6)}

Il appartient à l'auteur d'informer de l'existence de directives anticipées. Il peut le faire par oral, par exemple lors de son admission à l'hôpital, mais également à 
l'aide d'un document attestant de l'existence de directives anticipées ou, à l'avenir, en mentionnant leur existence sur la carte d'assuré. Cependant, le médecin devrait également toujours demander à son patient s'il a rédigé des directives anticipées.

Les directives anticipées peuvent être portées sur soi, déposées chez le médecin de famille ou le représentant thérapeutique ou remis à un dépositaire.

Lorsque le médecin est informé de l'existence de directives anticipées et du lieu de leur dépôt, il doit les réclamer.

\section{Que faire, lorsque le médecin, le représentant thérapeutique ou les proches ne parviennent pas à un accord? (chapitre 10)}

Les directives anticipées doivent être adaptées à la situation concrète. Il peut arriver que les avis des représentants thérapeutiques, des membres de l'équipe soignante et des proches divergent. Dans ce cas, il est souhaitable de recourir à une consultation d'éthique. Si celle-ci ne permet pas de trouver un accord, il convient de faire appel à l'autorité légale compétente (l'autorité tutélaire; dès l'entrée en vigueur de la nouvelle loi sur la protection de l'adulte: l'autorité de protection de l'adulte).

\section{Questions types concernant l'échelle des valeurs}

Les questions suivantes ont été formulées dans le but d'établir aussi précisément que possible l'échelle des valeurs du patient («anamnèse des valeurs»). Elles peuvent être intégrées à un entretien ou bien l'auteur des directives anticipées peut y répondre directement. Il est important que les réponses reflètent la situation de vie actuelle du patient. L'anamnèse des valeurs ne reflète donc toujours que le moment présent; elle peut évoluer au fil du temps et doit, le cas échéant, être réactualisée.

La description de l'échelle personnelle des valeurs est significative lors de la rédaction de directives anticipées, car elle permet de connaître les conceptions de vie, les craintes, les valeurs et les attentes qui sont déterminants pour le patient. Ces indications sur l'échelle des valeurs permettent d'avoir des repères lorsque le patient n'a pas apporté de précisions relatives à des mesures médicales spécifiques (chapitre 4.1.).

\section{La motivation}

Pour quelle raison souhaitez-vous rédiger des directives anticipées? Est-ce suite à un événement concret? Quelle est la finalité de ces directives anticipées resp. que voulez-vous éviter? En avez-vous discuté avec vos proches? Avec votre médecin de famille?

\section{La vie entre la naissance et la mort}

Où vous voyez-vous dans votre vie? Est-il important pour vous de vivre encore longtemps? Seriez-vous prêt, pour gagner des années de vie, à accepter certaines limitations (un état de dépendance, par exemple)? Ou bien préférez-vous renoncer à vivre quelques années de plus pour rester aussi indépendant que possible? Que signifie pour vous personnellement et concrètement «mourir dans la dignité»? Quel est le rôle de vos proches/de votre famille: quelles tâches êtes-vous prêt à assumer et que peut-on vous demander?

\section{La qualité de vie}

Qu'est-ce qui donne du sens à votre vie? Quelles activités, contenus et valeurs déterminent actuellement votre vie? Pensez-vous que la maladie ou un âge avancé puisse changer votre conception de la qualité de vie (par exemple en termes de capacité de communication, de mobilité, d'état d'esprit)? A quel point estil important pour vous de vivre sans douleur? Seriezvous prêt à accepter pour cela un état de conscience diminué voire même, dans le cas extrême, une perte de conscience?

\section{Expérience de la maladie, de la fin de vie et de la mort}

Avez-vous personnellement déjà vécu l'expérience de la maladie? Ou l'avez-vous vécu à travers un tiers (par exemple, parents, conjoint, ami)? De quelle façon ces expériences ont-elles influencé votre relation à la médecine, aux soins et à l'acceptation des prestations médicales? Vivez-vous actuellement avec des limitations ou des maladies? Voyez-vous un sens à la vie, si vous deviez subir des limitations sévères ou si votre personnalité devait changer (comme par exemple dans des cas de comas ou de démences sévères)? Pour survivre à une telle situation, seriez-vous prêt à accepter des inconforts liés à des traitements médicaux? Quels dommages ou préjudices considérez-vous comme assez graves pour ne plus vouloir continuer à vivre?

\section{Convictions personnelles et religieuses}

Avez-vous des convictions religieuses, spirituelles ou philosophiques; faites-vous partie d'une église ou d'une association? Ces convictions ont-elles une influence sur votre vie dans des «situations limites», c'est-à-dire quand votre vie est en danger (maintenir la vie ou laisser mourir). Y a-t-il des rituels ou d'autres éléments qui, en raison de vos convictions philosophiques ou religieuses, devraient être respectés après votre mort (utilisation du corps etc.)? Seriez-vous d'accord de donner vos organes ou tissus après votre mort (don d'organes)?

Le texte intégral des directives et recommandations «Directives anticipées» peut être consulté en ligne sous www.samw.ch/fr/Ethique/Directives/ actualite.html et commandé au Secrétariat général de I'Académie Suisse des Sciences Médicales (ASSM), Petersplatz 13, 4051 Bâle. 